\title{
Renal ultrasound: analysis and use in pregnant
}

\begin{abstract}
Authors renal biometry in pregnant women standardize without previous renal pathology findings reporting of renal Measures during gestation; Present report findings in renal registers pregnant Women with medical pathology associated renal disease except. A descriptive, correlational and prospective study is reported. The sample was not probabilistic and by intentional selection of 198 pregnant Patients, divided in group A of 149 Patients without associated medical conditions, and group B of 49 Patients Attended on Ultrasound Service on Maternal Fetal "Concepcion Palacios" Maternity Caracas Venezuela. A renal biometry percentiles scale for pregnant women during gestation was performed. Mean age of $24.2 \pm 6.2$ years for group A, and 26.2 \pm 7.1 years for group B Patients was registered. Data Obtained from renal biometry percentiles was used to build scales and to elaborate tables of renal pregnant women diameters. Findings of ultrasound measurement of renal morphology in pregnant women during the three trimester's pregnancy are Reported.
\end{abstract}

Volume 4 Issue 5 - 2018

\author{
Ginna Velasco,' Luanys Rivera,' Carlos \\ Cabrera,' Alexandra Rivero, I Trina Navas, ${ }^{2}$ \\ Carlos Contreras ${ }^{3}$ \\ 'Maternal-Fetal Medicine Service, Concepción Palacios \\ Maternity, Venezuela \\ ${ }^{2}$ Internal Medicine, Western General Hospital,Venezuela \\ ${ }^{3}$ Department of Pediatrics, Western General Hospital,Venezuela
}

Correspondence: Ginna Karolina Cabrera Velasco, Caracas, Venezula, Email: ginnavelasco@hotmail.com

Received: June 17, 2018| Published: October 02, 2018

Keywords: renal ultrasound, kidney diseases in pregnancy, renal ecobiometría

\section{Summary}

Biometrics renal standardizes in the gestating without pathology because there are no local measurements with these parameters during pregnancy in the country. The presence of renal disorders in pregnant women with medical conditions associated is evaluated. A descriptive, prospective and correlational study review. The sample is non-probabilistic type and intentional selection consisting of 198 pregnant patients, consisting of those who attended the consultation ultrasound of Maternal Fetal Medicine Maternity "Concepcion Palacios", which were distributed in a group of 149 pregnant women had no medical pathologies associated, and a B group of 49 pregnant women if presented. A table of percentiles for renal biometry pregnant was developed by gestational age. Ages ranged from $24.2 \pm 6,2$ years and 26.2 \pm 7.1 years for groups A and B, respectively. Primigestas predominated with $36.2 \%$ in group A and $42.8 \%$ in the group B. percentiles are calculated and the corresponding tables of renal diameters pregnant were prepared by gestational age. The sonographic findings reveal that diameters of renal morphology in pregnant women without renal disease in each of the trimesters of pregnancy.

\section{Introduction}

Pregnancy is a physiological event that has an impact on multiple organ systems. The kidneys are not exempt from these changes, so their structures are also affected by the growth of the gravid uterus and other own physiological changes of pregnancy. ${ }^{1}$ The most important modification is the dilation of the collecting, calyx, renal pelvis and ureter system, which may persist until the end of the puerperium; influenced by the relaxing action of progesterone on smooth muscle. Kidney increases $1 \mathrm{~cm}$ length, renal pelvis expands to $60 \mathrm{~mm}$ and ureters also dilate (most right) with a pronounced curvature. ${ }^{1-6}$ These structural changes may affect renal function, especially in the case of previous pathology of that body. ${ }^{1,2}$ The presence of associated medical conditions such as hypertensive and metabolic disorders in pregnancy and immunological diseases can also affect renal function. A helpful tool for monitoring kidney function is creatinine levels, blood urea nitrogen (BUN) and blood uric acid, as well as the examination of urinary sediment. Creatinine clearance and clearance within 24 hours measured in blood and urine are also supportive. ${ }^{6-10}$ Another useful tool is renal ultrasound evaluation that besides being simple to perform, safe and economical can be repeated as needed. ${ }^{10-14}$

There is currently no standardization in maternal renal biometry, allowing serve as a reference for comparing cutoffs according to gestational age and renal sonographic findings at the time of the evaluation, so that the realization of a renal ultrasound examination arises in pregnancy Normal, in order to set up their tables measures during pregnancy and determine the proper physiological changes of pregnancy and differentiate pathological through renal ultrasonography. There is no national literature, which have been reported studies on renal ultrasonography in pregnant women. In the literature review we did not work on nomograms renal biometry found in pregnant women. ${ }^{3}$ So the motivation to perform biometry renal nomograms arose during pregnancy. ${ }^{15-20}$

\section{Methods}

A descriptive, prospective and correlational study, whose population was integrated with pregnant women who attended sonographic evaluation at the Department of Ultrasonography Unit Maternal-Fetal Medicine Maternity Concepcion Palacios, during May 2014 to November 2014 was developed with approval of informed consent. The inherent data variables were collected from medical records. For the study included two ultrasound equipment, translators 3.5 Mhz-dimensional. Service specialists participated in Ultrasonography, consisting of a Perinatologist, two specialists in maternal-fetal medicine and one in Imaging. ${ }^{20-24}$

The sample consisted of 198 patients distributed in Group A 149 patients not recorded medical pathologies associated and Group B with 49 patients if they do, except chronic renal disease. For kidney measures values of longitudinal, transverse and anteroposterior 
diameters, thickness of the cortex, medulla and renal pelvis, both of the right kidney and the left were recorded, but only the measurements obtained in healthy pregnant women were used for the corresponding to the percentiles with those who were prepared calculation tables. It should be noted that the renal pelvis was not visible in most of the observations made, so it was not possible to establish valid measurements for inclusion in Table 1.

In the group with associated pathologies those presented ultrasonographic changes which may occur during pregnancy commonly included; however, cases were excluded sonographic findings indicative of kidney, such as polycystic kidney disease, renal agenesis, lithiasis, renal and adrenal tumors pathologies. ${ }^{25-28}$

The average and standard deviation of continuous variables were calculated. In the case of nominal variables frequencies and percentages calculated. Data were analyzed with SAS-JMP 11.0. Once identified patients without renal impairment was possible to develop percentile tables for renal biometry pregnant and gestational age was also associated with those ultrasonographic changes and associated pathology.

Table I Biometrics left kidney ultrasound in pregnant women without renal pathology according trimester (measured in mm., Average $\pm D E^{*}$ )

\begin{tabular}{llllll}
\hline \multicolumn{5}{c}{ Left kidney } \\
\hline & D. Long.** & AP D.*** & D. Transv.***** & Cortex & Marrow \\
First Quarter & $97.9 \pm 6.2$ & $50.9 \pm 5.2$ & $67.2 \pm 9.2$ & $19.0 \pm 3.0$ & $15.7 \pm 2.7$ \\
II Quarter & 101.9 & $53.1 \pm 8.2$ & $67.8 \pm 5.2$ & $19.9 \pm 3.0$ & $16.0 \pm 2.8$ \\
Third Quarter & 100.7 & $53.2 \pm 6.8$ & $65.6 \pm 6.6$ & $20.2 \pm 2.6$ & $16.6 \pm 2.4$ \\
\hline
\end{tabular}

*SD, standard deviation; **D Long, longitudinal diameter; ***DAP, diameter anteroposterior; ****D Transv, transverse diameter

Table 2 Biometrics right kidney ultrasound in pregnant women without renal pathology according trimester (measured in mm.,Average \pm DE*)

\begin{tabular}{llllll}
\hline \multicolumn{5}{c}{ Right kidney } \\
& D. Long.** & AP D.*** & D. Transv.**** & Cortex & Marrow \\
First Quarter & $97.6 \pm 7.9$ & $51.4 \pm 5.2$ & $66.2 \pm 5.0$ & $19.5 \pm 3.4$ & $16.3 \pm 3.4$ \\
II Quarter & 102.6 & $50.4 \pm 5.0$ & $66.9 \pm 6.8$ & $20.4 \pm 2.6$ & $16.9 \pm 2.5$ \\
Third Quarter & 101.2 & $51.6 \pm 8.5$ & $68.22 \pm 8.1$ & $20.5 \pm 3.1$ & $16.4 \pm 2.8$ \\
\hline
\end{tabular}

*SD, standard deviation; **D. Long, longitudinal diameter; ***D. AP, diameter anteroposterior; ****D Transv, transverse diameter

Table 3 Distribution of medical conditions associated according to the trimester

\begin{tabular}{llll}
\hline Trimester & & & \\
\hline Pathology & I & II & III \\
Urinary tract infection & 5 & 6 & 6 \\
Chronic artery hypertension & 8 & 4 & 3 \\
hypertensive disorder of pregnancy & - & 2 & 4 \\
Mellitus diabetes & 2 & 3 & - \\
Hypo- or hyperthyroidism & I & 2 & I \\
Systemic lupus erythematosus & I & - & - \\
Rheumatoid arthritis & - & - & I \\
total & I7 & I7 & fifteen \\
\hline
\end{tabular}


Table 4 Ratio ultrasonographic changes with medical conditions associated

\begin{tabular}{|c|c|c|c|c|c|c|}
\hline sonographic findings & And You & The & DM & tiroide & Them & Total \\
\hline Increased birefringence in the cortex & & 6 & 1 & & 1 & 8 \\
\hline Refringencia decreased in the cortex & 2 & & & & & 2 \\
\hline microlitiasis & 3 & & & & & 3 \\
\hline pyelectasis & & I & & & & 1 \\
\hline hydronephrosis & 5 & & & & & 5 \\
\hline ureterohydronephrosis & 2 & & & & & 2 \\
\hline Total & 12 & 7 & 1 & & I & twenty-one \\
\hline
\end{tabular}

\section{Discussion}

In national medical literature no publications on renal ultrasonographic measurements in pregnant women, so the current report will serve as initial reference guide for possible kidney diseases in pregnant women. In analyzing the data obtained normal changes in renal function and anatomic variations that occur during pregnancy, both in the kidney and urinary tract, without posing pathological alterations, which result in modifications of its structure corroborate that they can be detected by ultrasound during pregnancy. This investigation identified 17 patients with urinary tract infection and 21 cases with concomitant ultrasonographic changes that were part of the group of pregnant associated pathology. The findings allow to demonstrate the usefulness of ultrasonography in patients with a history of recurrent urinary tract infections; and to monitor renal health of these patients during pregnancy, to identify the cause predisposing to infection may be silent. ${ }^{29,30}$

A high frequency of hypertension (15 patients) was also recorded, to which added 6 pregnant women with hypertensive disorders are associated with pregnancy. And 5 patients with Diabetes Mellitus, in which attention is not confined to pregnancy, but beyond, to offer alternatives and avoid severe health complications later in life. ${ }^{31}$ Other diseases related to autoimmune diseases, which is consistent with the age and merits multidisciplinary care ${ }^{32,33-35}$ Renal ultrasound measurements allowed for nomograms percentiles of renal parameters at different gestational ages, which aimed to identify associated medical conditions. For this reason it is recommended routine procedure in all patients with a history of recurrent pathologies associated with this body, in an attempt to pesquisar early cases will generate complications

\section{Acknowledgments}

None.

\section{Conflicts of interest}

The author declares there are no conflicts of interest.

\section{References}

1. Cabero L. Renal diseases and urinary system during pregnancy. Obstetrics and Maternal-Fetal Medicine. Panamericana. Madrid, Spain; 2007:231-235.

2. Dunlop W. Renal physiology in pregnancy. Postgrad Med J. 1979;55(643):329-332.

3. Haunch D, Chain, L. Renal fetal ultrasound biometry. Maternity "Concepción Palacios". Thesis for specialist degree. Venezuela; 2004.
4. Toirac A, Pascual V. The kidney and urinary excretory system in pregnant. Basic considerations. Medisan. Madrid, Spain; 2013. p. 68-79.

5. Dafnis E, Sabatin S. The effect of pregnancy on renal function; Physiology and Pathophysiology. Am J Med. 1992;303(3):184-205.

6. J Gallo M. Padilla renal function in pregnancy. Clinical and research Gynecology and Obstetrics. Madrid, Spain; 2000.

7. Gleicher N, Urinary tract infections in pregnancy. Clinical Medicine in Obstetrics. Panamericana. Buenos Aires, Argentina; 1994. p. 54-59.

8. Chapman A, Zamodia S, Osorio F. Systemic and renal hemodynamic changes in the phase of the menstrual luthéal mime cycle early pregnancy. Am J Physiol. 1997;4(3):24-29.

9. Vigil P, Gallo M, Espinoza A. High-risk pregnancy and its complications. Amolca, Venezuela; 2011:81-93.

10. Rivera M, Quevedo C. Sonographic by nephrologists. Nefroplus. 2009;2(1):9-16.

11. Liu KD, Chertow GM. Curbing the use of ultrasonography in the diagnosis of acute kidney injury. Arch Intern Med. 2010;170(21):19071908.

12. Licurse A, Dziura J. Renal ultrasonography in the evaluation of Acute Kidney Injury. Arch Intern Med. 2010;170(21):1901-1907.

13. Krane NK. Acute renal failure in pregnant. Rev Med Int. 1998;148(11):2347-2357.

14. Martinez R, Herrera J. Nephrology Treaty. Urinary infections. Norma. Madrid, Spain; 1993. p. 124-129.

15. Reyes P. Nephropathy and pregnancy. Perinatol Rev. 1995;10(3):1118.

16. Natterson J, Beiko D. Ureteral calculi for Management strategy in pregnancy. Urology. 2002;60:383.

17. Sturgiss S, Wilkinson R, Davison J. Renal haemodynamics and tubular function in human pregnancy. Am J Physiol. 1996;271(2):16-20.

18. L Diaz. Risk of anesthesia in renal function. Rev Mex Anest. 2005;28:233-238.

19. Arias F. Kidney diseases during pregnancy. Practical Guide to pregnancy and childbirth high risk. Harcourt Brace; 1995:47-83.

20. Avechua ZR. Blas renal abscess in pregnancy. Rev Mex Urol. 2012;72(6):310-313.

21. Kes P, Basić Jukić N. Acute Kidney Injury in the intensive care unit. Crit Care Med. 2007;35:1837-1843.

22. Pastore A. Ultrasonography of Gynecology and Obstetrics. Fetal malformations urinary tract. Cerri Amolca G, editor. Venezuela; 2012 p. 363-383. 
23. M Foley F. Garite in Intensive Care Obstetrics. Acute renal failure in pregnancy. Amolca, Venezuela; 2011:165-173.

24. Duran C, Reyes N. kidney disease and pregnancy. Rev Hosp Gral. 2006;7(2):82-89.

25. Gratacos E, Gómez R. Nephrourological Malformations. Fetal medicine. Panamericana. Madrid, Spain; 2009:427-439.

26. Seidman D, Soriano D. Role of renal ultrasonography in the management of pregnant women in pyelonephritis. $J$ Perinatal. 1998; $18: 98$

27. Jungers PD. Chauran renal disease in Pregnancy. Kidney Internat. 1997;52:871-885.

28. Jungers P, P Houillier, Forget D, et al. Specific Controversies Concerning the natural history of renal disease in pregnancy. $\mathrm{Am} \mathrm{J}$ Kidney Dis. 1991;17:116-122.

29. Wiles K, Bramham K, Vais A, et al. Pre-pregnancy counseling for Women with chronic kidney disease: a Retrospec-tive analysis of nine years' experience. BMC Nephrol. 2015;16:28.

30. Chen S, Sun X, Wu B, et al. Pregnancy in Women with Systemic Lupus Erythematosus: A retrospective study of 83 pregnancies at a single Center. Int J Environ Res Public Health. 2015;12(8):9876-9888.

31. Singh R, Prasad N, Banka A, et al. Pregnancy in Patients With chronic kidney disease: maternal and fetal outcomes. Indian J Nephrol. 2015;25(4):194-199.

32. Guenter S. Differential Diagnosis in Ultrasonido. Cap 8 Kidneys. Amolca. Venezuela; 2010:249-272.

33. Berthold B. Abdominal ultrasound. Kidneys. Panamericana Editorial, Madrid Spain; 2011:191-229.

34. Mathias Hofer. Basic Course sonography. Kidneys. Panamericana Editorial. 2000:45-62.

35. Fescine R, Martinez E. Monitoring fetal growth. Newsletter CLAP / WHO. Uruguay; 2013. 